\title{
Electrically transmissive alkyne-anchored monolayers on gold
}

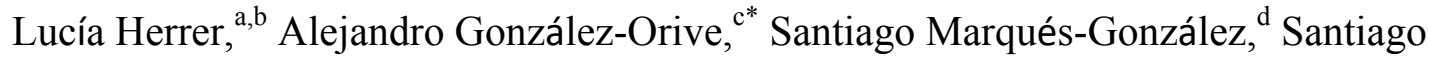

Martín, ${ }^{\mathrm{a}, \mathrm{e}}$ Richard J. Nichols, ${ }^{\mathrm{f}}$ José Luis Serrano, ${ }^{\mathrm{a}, \mathrm{b}}$ Paul J. Low, ${ }^{\mathrm{g}}$ Pilar Cea, ${ }^{\mathrm{a}, \mathrm{b}, \mathrm{e}^{*}}$

[a] Departamento de Química Física, Facultad de Ciencias, Universidad de Zaragoza, 50009, Zaragoza, Spain.

[b] Instituto de Nanociencia de Aragón (INA) and Laboratorio de Microscopias Avanzadas (LMA), edificio i+d Campus Río Ebro, Universidad de Zaragoza, C/Mariano Esquillor, s/n, 50018, Zaragoza, Spain.

[c] Technical and Macromolecular Chemistry, University of Paderborn, Warburger Strasse 100, 33098 Paderborn, Germany.

[d] Department of Chemistry, Durham University, South Rd, Durham, DH1 3LE, UK.

[e] Instituto de Ciencias de Materiales de Aragón (ICMA), Universidad de ZaragozaCSIC, 50009, Zaragoza, Spain.

[f] Department of Chemistry, University of Liverpool, Crown Street, Liverpool, L69 7ZD, United Kingdom.

[g] School of Molecular Sciences, University of Western Australia, 35 Stirling Highway, Crawley, Perth, 6009, Australia.

Corresponding authors:

Alejandro González-Orive (agorive@mail.upb.de)

Pilar Cea (pilarcea@unizar.es) 


\begin{abstract}
Well-ordered, tightly-packed (surface coverage $0.97 \times 10^{-9} \mathrm{~mol} \cdot \mathrm{cm}^{-2}$ ) monolayer films of 1,4-bis((4-ethynylphenyl)ethynyl)benzene (1) on gold are prepared via a simple self-assembly process, taking advantage of the ready formation of alkynyl $\mathrm{C}$-Au $\sigma$-bonds. Electrochemical measurements using $\left[\mathrm{Ru}\left(\mathrm{NH}_{3}\right)_{6}\right]^{3+},\left[\mathrm{Fe}(\mathrm{CN})_{6}\right]^{3-}$, and ferrocenylmethanol $\left[\mathrm{Fe}\left(\eta^{5}-\mathrm{C}_{5} \mathrm{H}_{4} \mathrm{CH}_{2} \mathrm{OH}\right)\left(\eta^{5}-\mathrm{C}_{5} \mathrm{H}_{5}\right)\right]$ redox probes indicate that the alkynyl $\mathrm{C}-\mathrm{Au}$ contacted monolayer of 1 presents a relatively low barrier for electron transfer. This contrasts with monolayer films on gold of other oligo(phenylene ethynylene) derivatives of comparable length and surface coverage, but with different contacting groups. Additionally, a low voltage transition $\left(V_{\text {trans }}=0.51 \mathrm{~V}\right)$ from direct tunneling (rectangular barrier) to field emission (triangular barrier) is observed. This low transition voltage points to a low tunneling barrier, which is consistent with the facile electron transport observed through the C-Au contacted self-assembled monolayer of $\mathbf{1}$.
\end{abstract}




\section{TOC GRAPH}

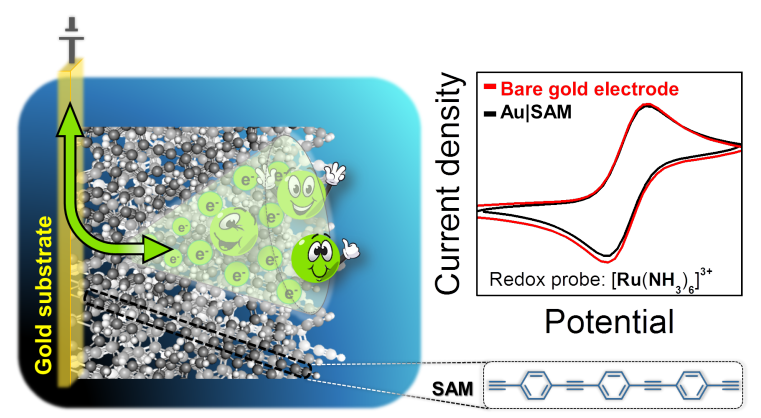




\section{Introduction}

Surface-molecule interactions underpin the development of surface-functionalised clusters, metal nanoparticles (NPs) and electrodes and such systems have great promise in areas including microelectronics, microelectromechanical systems, molecular electronics, surface protection, sensing, electrochemistry, electrocatalysis, plasmonics and drug delivery systems, to name but a few. ${ }^{1-9}$ Thiols (as thiolates) have been widely used as capping agents for metal (particularly gold and silver) nanoparticles, and as the exemplary contacting group in exploratory molecule-metal (gold) junctions for molecular electronics. ${ }^{10,} 11$ However, despite the strength of the Au-S bond and the ease of fabrication of thiol-on-gold single-molecule contacts and SAMs, the fluxional nature of the Au-S bond coupled with oxidation, thermal instability, and degradation of thiolate-contacted molecules on metals in aqueous and ambient environments represent an impediment to their wider application. Other functional groups, including amines, ${ }^{12}$ carboxylic acids, ${ }^{12-14}$ dithiocarboxylic acids, ${ }^{15}$ esters, ${ }^{16}$ pyridyl, ${ }^{17-20}$ cyano, ${ }^{21,} 22$ isocyano, ${ }^{23,}{ }^{24}$ isothiocyanate,,${ }^{25}$ nitro, ${ }^{21}$ methylselenide, ${ }^{26}$ methylthiol, ${ }^{26}$ dimethylphosphine, ${ }^{26}$ ethynyltrimethylsilane, ${ }^{27-33}$ viologens $^{34}$ and fullerenes ${ }^{13,35}$ have been studied as potential molecule-contacting groups for various metal surfaces. However, these metal|molecule contacts are also characterized by fluxional bonds to the surface leading to structural rearrangements of the films, surface etching and reforming, and, in the case of molecular electronic studies, the observation of multiple conductance signals, stochastic on-off switching of the molecule-metal contact, high

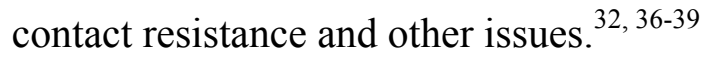

In recent years, robust molecule-on-gold contacting with direct $\mathrm{Au}-\mathrm{C}^{19,40-45}$ anchoring has been achieved, and in studies to date molecular junctions formed with these contacts have proven to be free of many of the complications noted above, prompting further exploration of these systems. Our group has recently described the formation of a sandwiched Au-Cmolecular wire-C-Au device, with the molecular wires bridging a gold electrode bottom contact and a gold nanoparticle top contact. In this earlier work, the Au-C contacting was achieved by sequential fluoride-induced desilylation/metalation of a trimethylsilylprotected organic moiety, ${ }^{44,46}$ with the formation of Au-C $\sigma$-bonds at both the bottom and the upper electrodes. ${ }^{47}$ The main objective of this previous work was to prepare "all carbon" molecular bridges comprised of a $-\mathrm{C} \equiv \mathrm{C}-\mathrm{C} \equiv \mathrm{C}-\mathrm{C} \equiv \mathrm{C}-\mathrm{C} \equiv \mathrm{C}$ - chain and to probe the 
integrity of the device chemically bonded between the two electrodes. This present contribution extends this work in two ways by demonstrating (i) the straightforward formation of a self-assembled monolayer film of the oligo(phenylene ethynylene) (OPE) derivative 1,4-bis((4-ethynylphenyl)ethynyl)benzene (1) (Figure 1) with direct activation of a terminal ethynyl moiety on an $\mathrm{Au}(111)$ electrode to form the top contact, thereby dispensing with the manipulation of the TMS group during formation of the surface contact;(ii) by in contrast to OPE-monolayers contacted to gold electrodes by groups such as thiols, amines, acids, etc., the Au-C contacted monolayers of $\mathbf{1}$ effectively mediate electron transfer (ET) between redox active molecules in solution and the electrode in electrochemical experiments. $^{40,46,48,49}$

Compound 1

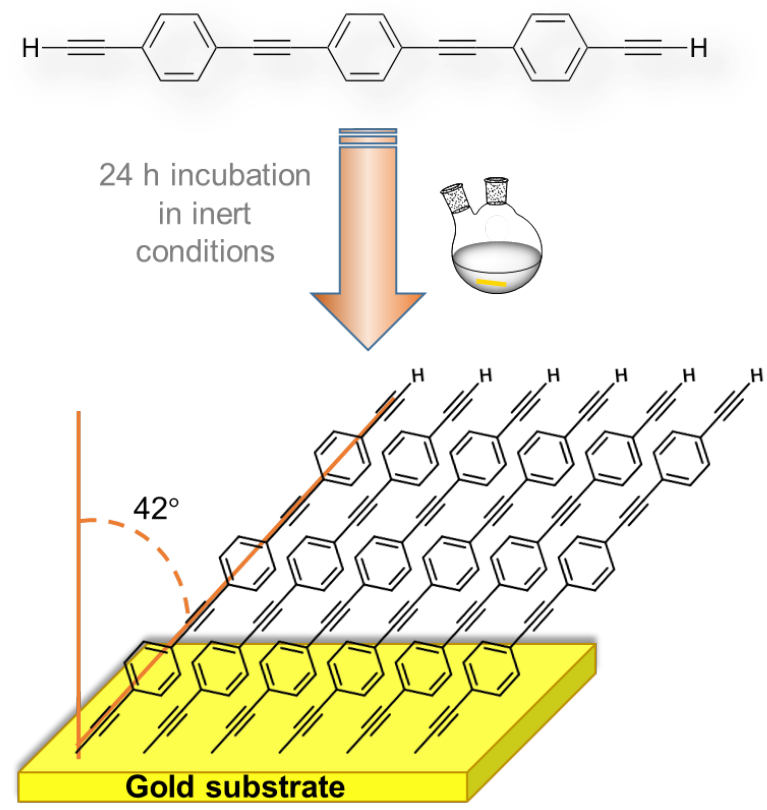

Figure 1. Cartoon showing the molecular structure of 1,4-bis((4ethynylphenyl)ethynyl)benzene, compound $\mathbf{1}$, and the incubation process leading to highly compact monolayers where the molecules exhibit a tilt angle of $42^{\circ}$ with respect to the normal surface.

\section{Experimental}

All reagents were purchased from Sigma-Aldrich (now Merck) or prepared using minor modifications or the literature methods as cited below. Reactions were carried under 
an atmosphere of nitrogen using standard Schlenk techniques, although no special precautions were employed during conventional work-up procedures.

Synthesis of 1. A Schlenk flask was charged with dried, degassed $\mathrm{NEt}_{3}(20 \mathrm{~mL})$, to which $\mathrm{Me}_{3} \mathrm{SiC} \equiv \mathrm{CC}_{6} \mathrm{H}_{4} \mathrm{C} \equiv \mathrm{CH}(0.37 \mathrm{~g}, 1.5 \mathrm{mmol})$, 1,4-diiodobenzene (0.23 g, $\left.0.70 \mathrm{mmol}\right)$, $\mathrm{Pd}\left(\mathrm{PPh}_{3}\right)_{4}(0.09 \mathrm{~g}, 0.10 \mathrm{mmol})^{50}$ and $\mathrm{CuI}(0.02 \mathrm{~g}, 0.10 \mathrm{mmol})$ were added and the resulting suspension stirred at room temperature overnight. The precipitate was collected by filtration and washed thoroughly with hexane. The product was then recrystallized from toluene, yielding 1,4-bis((4-trimethylsilylethynylphenyl)ethynyl)benzene as white needles (0.13 g, $0.28 \mathrm{mmol}, 40 \%)$. [ ${ }^{1} \mathrm{H}$ NMR (400 MHz, $\mathrm{CDCl}_{3}$ ) $\delta: 7.50$ (s, 4H), 7.45 (s, br, 8H), 0.26 (s, 18H). ${ }^{13} \mathrm{CNMR}\left(101 \mathrm{MHz}, \mathrm{CDCl}_{3}\right) \delta: 132.1,131.7,131.6,123.4,123.2,123.3,104.8,96.6$, 91.2, 91.1, 0.1. $\mathrm{MS}^{+}$(ASAP) $\left.m / z(\%): 470.20\left(100,[\mathrm{M}]^{+}\right)\right]^{51}$ A solution of 1,4-bis((4trimethylsilylethynylphenyl)ethynyl)benzene $(0.05 \mathrm{~g}, 0.11 \mathrm{mmol})$ in THF $(35 \mathrm{~mL})$ was treated with a solution of $\mathrm{K}_{2} \mathrm{CO}_{3}(0.04 \mathrm{~g}, 0.29 \mathrm{mmol})$ in methanol $(5 \mathrm{~mL})$ and the mixture allowed to stir overnight. The solvent was removed under reduced pressure and the residue re-dissolved in $\mathrm{CHCl}_{3}(50 \mathrm{~mL})$. The solution was washed with water $(2 \times 50 \mathrm{~mL})$, brine (1 x $25 \mathrm{~mL}$ ) and the organic layer dried over $\mathrm{MgSO}_{4}$. The solvent was removed giving 1 as a rather insoluble white powder $(0.029 \mathrm{~g}, 0.088 \mathrm{mmol}, 81 \%) .{ }^{1} \mathrm{H} \mathrm{NMR}\left(400 \mathrm{MHz}, \mathrm{CDCl}_{3}\right) \delta$ : $7.51(\mathrm{~s}, 4 \mathrm{H}), 7.48(\mathrm{~s}, 8 \mathrm{H}), 3.18(\mathrm{~s}, 2 \mathrm{H}) \cdot \mathrm{MS}^{+}$(ASAP) $\left.\mathrm{m} / z(\%): 326.1\left(100,[\mathrm{M}]^{+}\right)\right]$, Figure $\mathrm{S} 1$ in the Supporting information, SI. IR (Nujol): $3271 \mathrm{~m}, v(\equiv \mathrm{C}-\mathrm{H}){ }^{52}$

SAMs were prepared under an oxygen free environment using standard Schlenk techniques $^{53,54}$ by immersion of a clean gold electrode (prepared as described below for each method of measurement) within a freshly prepared $1 \cdot 10^{-4} \mathrm{M}$ solution of $\mathbf{1}$ in a deoxygenated $2 \cdot 10^{-4} \mathrm{M} \mathrm{NEt}_{3}$ solution in $\mathrm{CHCl}_{3}$ (HPLC grade, 99.9\% degassed by three freeze pump-thaw cycles), and stirred overnight at $45^{\circ} \mathrm{C}$ under an argon atmosphere. After reaction, the substrate was removed and rinsed with copious amounts of $\mathrm{CHCl}_{3}$ to remove physisorbed material, and dried under a stream of $\mathrm{N}_{2}$. The as prepared samples were stored under inert atmosphere before use.

Quartz Crystal Microbalance (QCM) measurements were carried out using a Stanford Research System instrument employing AT-cut, $\alpha$-quartz crystals with a resonant frequency of $5 \mathrm{MHz}$ having circular gold electrodes patterned on both sides. The gold substrates were used for the first time for this experiment and they were just cleaned with 
ethanol and allowed to dry. Contact angle measurements were performed with a commercial optical tensiometer (Theta Lite from Attension).

Raman and Surface-enhanced Raman scattering (SERS) spectra were recorded using a Confocal Raman Imaging purchased from Witec (model Alpha300M+) with an excitation wavelength of $633 \mathrm{~nm}$. SERS substrates were prepared using a previously described methodology. ${ }^{55}$ Briefly, a $\mathrm{SiO}_{2}$ wafer was incubated in a poly(diallyldimethilammonium) chloride aqueous solution (PDDA), $0.2 \% \mathrm{v} / \mathrm{v}$ for 4 hours; followed by rinsing with deionized water and drying under a $\mathrm{N}_{2}$ stream. The substrate was subsequently immersed in gold nanoparticle solution (synthesized via a modified version of Turkevich method) for 12 hours at $4{ }^{\circ} \mathrm{C}$; afterwards, the SERS substrates were washed with deionized water and $\mathrm{N}_{2-}$ dried. Finally, $\mathrm{O}_{2}$-plasma treatment was performed to clean the SERS substrate surface (conditions: 0.2 mbar, $50 \mathrm{~W}$ and $60 \mathrm{~s}$ ). AFM images were obtained in tapping and PeakForce modes using a Multimode 8 microscope equipped with a Nanoscope $\mathrm{V}$ control unit from Bruker operating in ambient air at a scan rate of 0.5-1.2 Hz, using RFESPA-75 (75$100 \mathrm{kHz}$, and $1.5-6 \mathrm{~N} \cdot \mathrm{m}^{-1}$, nominal radius of $\left.8 \mathrm{~nm}\right)$ and ScanAsyst-Air-HR (130-160 kHz, and $0.4-0.6 \mathrm{~N} \cdot \mathrm{m}^{-1}$, nominal radius of $2 \mathrm{~nm}$ ) cantilever/tip assemblies, purchased from Bruker. Root Mean Squared (RMS) roughness, Bearing and Depth statistical analysis were carried out off-line with Nanoscope v. 1.40 and Gwyddion v. 2.41 software packages.

An Agilent 5500 SPM microscope (Agilent Picoscan 5.3.3 software) was used for determining the electrical properties of the SAM with the "STM touch-to-contact" method. ${ }^{56}$ STM tips were freshly prepared for each experiment by etching of a $0.25 \mathrm{~mm} \mathrm{Au}$ wire $(99.99 \%)$ in a mixture of ethanol: $\mathrm{HCl} 37 \% 1: 1$ at $+2.4 \mathrm{~V}$. STM imaging was carried out by using a Nanoscope IIIa (Digital Instruments/Veeco) operating in air at room temperature. Pt-Ir tips purchased from Bruker were used in these measurements. Typical bias potentials, tunneling currents, and scan rates were in the range of 0.3-0.6 V, 0.2-0.5 nA, and 1-6 Hz, respectively. The surface coverage, surface features such as raised islands, vacancies and pits, as well as depth histograms, were assessed with the WSxM, Gwyddeon and Nanoscope software. Gold substrates were purchased from Arrandee ${ }^{\mathrm{Tm}}$, Germany and flame-annealed at approximately $800-1000{ }^{\circ} \mathrm{C}$ with a Bunsen burner immediately prior to use. This procedure is known to result in atomically flat $\mathrm{Au}(111)$ terraces. $^{57}$ 
X-ray photoelectron spectroscopy (XPS) spectra were recorded with a Kratos AXIS ultra DLD spectrometer incorporating a monochromatic Al K $\alpha$ X-ray source (1486.6 eV) using a pass energy of $20 \mathrm{eV}$. The photoelectron take-off angle was $90^{\circ}$ with respect to the sample plane. The XPS binding energies were referenced to the $\mathrm{Au}\left(4 \mathrm{f}_{7 / 2}\right)$ peak at $84.0 \mathrm{eV}$ in order to provide a precise energy calibration. The thickness of these SAMs on the gold substrates was determined from the attenuation of the Au-4f signal from the substrate according to the equation: $\mathrm{I}_{\mathrm{SAM}}=\mathrm{I}_{\text {substrate }} \exp (-\mathrm{d} / \lambda \sin \theta),{ }^{58}$ where $d$ is the SAM thickness, $I_{S A M}$ and $I_{\text {substrate }}$ are the average of the intensities of the $\mathrm{Au}\left(4 \mathrm{f}_{5 / 2}\right)$ and $\mathrm{Au}\left(4 \mathrm{f}_{7 / 2}\right)$ peaks attenuated by the SAM and from bare gold, respectively, $\theta$ is the photoelectron take-off angle, and $\lambda$ is the effective attenuation length of the photoelectron $(4.2 \pm 0.1 \mathrm{~nm}) .{ }^{59}$

Cyclic voltammetry (CV) experiments were performed with an Autolab potentiostat from Eco Chemie and a standard three-electrode cell. The working electrode was a gold substrate from Arrandee ${ }^{\mathrm{TM}}$ (either the bare gold electrode or the gold electrode covered by a SAM of 1) connected to the potentiostat by means of a cable terminated in a metallic crocodile clip that held the electrode. ${ }^{60}$ The reference electrode was $\mathrm{Ag} / \mathrm{AgCl}, \mathrm{KCl}(3 \mathrm{M})$, and the counter electrode was a Pt sheet.

\section{Results}

SAMs of 1 were prepared as indicated in the experimental section and Figure 1. Quartz Crystal Microbalance (QCM) studies indicate that the SAM of 1 exhibits a surface coverage of $0.97 \times 10^{-9} \mathrm{~mol} \cdot \mathrm{cm}^{-2}$ (Figure S2), which is typical of tightly-packed monolayers of OPE derivatives. ${ }^{20,61}$ Further evidence of the monolayer formation was obtained by measuring the contact angle of a drop of water onto a bare gold electrode $\left(39^{\circ}\right)$ and onto the same gold electrode after the deposition of the monolayer $\left(92^{\circ}\right)$. Large-area imaging of the modified surface using Atomic Force Microscopy (AFM) revealed a homogenous surface structure free of significant three-dimensional aggregates or large voids (Figure S3). By scratching the surface with the AFM tip and by carrying out a statistical analysis of the depths of the scratched region and surrounding areas, ${ }^{62,63}$ an average thickness of the SAM of 1 on the gold substrate of $1.7 \pm 0.2 \mathrm{~nm}$ was estimated. A very similar estimation of the film thickness, $1.7 \pm 0.1 \mathrm{~nm}$, was obtained by measuring the attenuation of the Au- $4 \mathrm{f}$ signal from the substrate in X-ray photoelectron spectroscopy experiments (XPS) after deposition 
of the SAM (Figure S4) as explained in the experimental section. Given the molecular length of 1 ( $2.3 \mathrm{~nm}$, estimated from computational molecular models: Spartan 08 V1.2.0), it can be concluded that within the film the molecules of 1 exhibit an average tilt angle of $42^{\circ}$ with respect to the surface normal to the electrode (Figure 1). Chemisorption of $\mathbf{1}$ onto the gold substrate and formation of a C-Au bond was demonstrated by comparing the Raman spectrum of the powder of $\mathbf{1}$ and the SERS spectrum of a monolayer of $\mathbf{1}$ onto gold (Figure S5). The SERS spectrum of the SAM of 1 shows a low intensity band at $418 \mathrm{~cm}^{-1}$, which provides a clear evidence of a covalent Au-C o-bond. ${ }^{64}$ Additionally, a broad band at 1981 $\mathrm{cm}^{-1}$ in the SERS spectrum of the SAM of $\mathbf{1}$ is also indicative of the formation of a $-\mathrm{C} \equiv \mathrm{C}$ Au bond. ${ }^{19}$ Further details of the Raman spectra can be found in the SI. In the majority of alkynyl monolayers on gold studied to date, the molecules have been found to be contacted to the gold surface as the acetylide after loss of the alkynyl $\equiv \mathrm{C}-\mathrm{H}$ proton, ${ }^{2}$ and oriented more or less normal to the substrate. ${ }^{43,54,65}$ However, DFT calculations suggest that this 'perpendicular' orientation is the minimum in a shallow potential energy surface, and that the total energy changes by less than $2 \mathrm{kcal} \cdot \mathrm{mol}^{-1}$ as the tilt angle increases to as much as $50^{\circ}$ with respect to the normal surface. ${ }^{66}$ The same authors have noted that the SAM structure will be strongly influenced by the interactions between the 'tails' of the alkyne moieties, on account of this low energy barrier for tilting the alkynyl backbone away from the surface normal. In the case of $\mathbf{1}$, it seems likely that the SAM is stabilized by dispersive $\pi-\pi$ interactions between the offset faces of the aromatic moieties. Such a staggering between adjacent aromatic rings would reasonably account for the overall tilt of molecules within the monolayer, and facilitated by the low energy barrier to tilting from the 'upright' position (Figure 1). Additional information about the organization of molecules of $\mathbf{1}$ within the film has been obtained from scanning tunneling microscopy (STM) and can be found in the SI (Figures S6-S12).

The identification of well-ordered and tightly packed SAMs of the 'wire-like' OPE derivative 1 on gold substrates prompted us to consider the electron-transfer behavior of these molecule-on-metal assemblies. By performing cyclic voltammetry in the presence of electrochemical redox probes, valuable information about the overall charge transfer processes through the SAM of $\mathbf{1}$ can be obtained. Depending on the shape, peak current intensities, and peak potential separations exhibited in the voltammograms, it is possible to 
identify and distinguish contributions to the faradaic current arising from electron tunneling through the organic layer or due to penetration of the redox-probe molecules through defects/pinholes in the SAM to the underlying metal electrode surface. ${ }^{67}$ Furthermore, redox probes can be classified according to the sensitivity of their electron transfer kinetics to the surface chemistry of the electrode. ${ }^{68}$ Heterogeneous outer-sphere redox systems, such as $\left[\mathrm{Ru}\left(\mathrm{NH}_{3}\right)_{6}\right]^{3+}$ and ferrocenylmethanol $\left(\mathrm{FcCH}_{2} \mathrm{OH}\right)$, are not especially sensitive to the nature of the electrode surface. ${ }^{69-71}$ In contrast, for systems that undergo heterogeneous inner-sphere electrode reactions at the electrode, specific interactions between the redox probe and the surface of the electrode or materials deposited on this surface occur during the electron transfer reaction. Therefore, the rate constant of a heterogeneous inner-sphere electrode reaction should be highly dependent on the electrode material and very sensitive to the surface state. The redox probe $\left[\mathrm{Fe}(\mathrm{CN})_{6}\right]^{3-}$ exhibits certain "inner-sphere" characteristics and a surface sensitive redox response. ${ }^{68,72,73}$ In the seminal work by Fleischmann et al., ${ }^{72}$ the adsorption of ferro/ferricyanide species on gold surfaces preceding and following electron transfer was elegantly demonstrated by carrying out in situ electrochemical SERS measurements. The authors claim structural changes in ferro/ferricyanide adsorbates depending on the applied potential and the nature of the support electrolyte. Either a coplanar arrangement of $\mathrm{C} \mathrm{N}$ groups (via $\pi^{*}$ orbital in the C N) on the $\mathrm{Au}(111)$ surface or a bridging bond ( $\sigma^{*}$ orbital) to the metal surface involving the lone pair of $\mathrm{N}$ atoms has been proposed to account for the molecule-surface interaction. Partial or complete surface blocking can therefore lead to significant and even total suppression of the electron transfer reactions of $\left[\mathrm{Fe}(\mathrm{CN})_{6}\right]^{3-}$ resulting in decreased peak currents and increases in the potential peak separation (i.e. blocking of faradaic reaction). Such behavior has been reported for gold electrodes modified by aryl-monolayer ${ }^{74,75}$ or by oligo(phenylene ethynylene) derivatives anchored through various groups including thiols, amines and carboxylic acids. ${ }^{12,14,31,60,61,76,77}$

The reversible two-peaked diffusion-controlled electrochemical response of the $\left[\mathrm{Ru}\left(\mathrm{NH}_{3}\right)_{6}\right]^{3+}$ electrochemical redox probe at a gold electrode modified by a SAM of $\mathbf{1}$ is almost indiscernible from that recorded using a bare gold electrode (Figure 2a). A similar result was achieved with $\mathrm{FcCH}_{2} \mathrm{OH}\left(\mathrm{Fe}\left(\eta^{5}-\mathrm{C}_{5} \mathrm{H}_{4} \mathrm{CH}_{2} \mathrm{OH}\right)\left(\eta^{5}-\mathrm{C}_{5} \mathrm{H}_{5}\right)\right)$, with little decrease in the faradaic current through the surface-modified electrode compared with the bare gold 
electrode (Figure 2b). Therefore on the timescale of these voltammetric determinations the monolayer of $\mathbf{1}$ is nearly transparent to the electron transfer process, which is in stark contrast with other monolayers of other OPE compounds that significantly or completely block the electrode. ${ }^{12,14,31,56,61,76,78}$ By comparison, in the case of the $\left[\mathrm{Fe}(\mathrm{CN})_{6}\right]^{3-}$ redox probe more changes are seen in the cyclic voltammetry when compared to the more straightforward outer-sphere redox species $\left(\left[\mathrm{Ru}\left(\mathrm{NH}_{3}\right)_{6}\right]^{3+}\right.$ and $\left.\mathrm{FcCH}_{2} \mathrm{OH}\right)$ studied in this work. A comparison of $\mathrm{CVs}$ of $\mathrm{K}_{3}\left[\mathrm{Fe}(\mathrm{CN})_{6}\right]$ recorded with pristine and modified electrodes demonstrates a more significant attenuation in the peak current pointing to a more significant, but far from complete, blocking of electron-transfer arising from the SAM of 1 (Figure 2c). For comparison purposes, when this redox probe is studied with a SAM of octadecanethiol (2) adsorbed on a gold electrode complete ET blocking is observed (Figure 2). It therefore appears that the SAM of $\mathbf{1}$ is an effective conduit of electron-transfer between the redox species in solution and the electrode, as demonstrated with the outersphere redox probe.

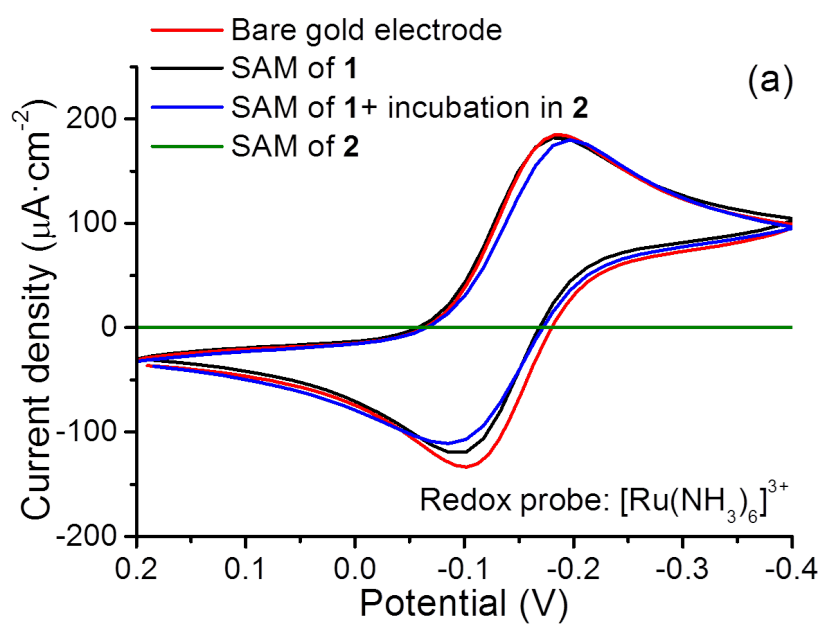



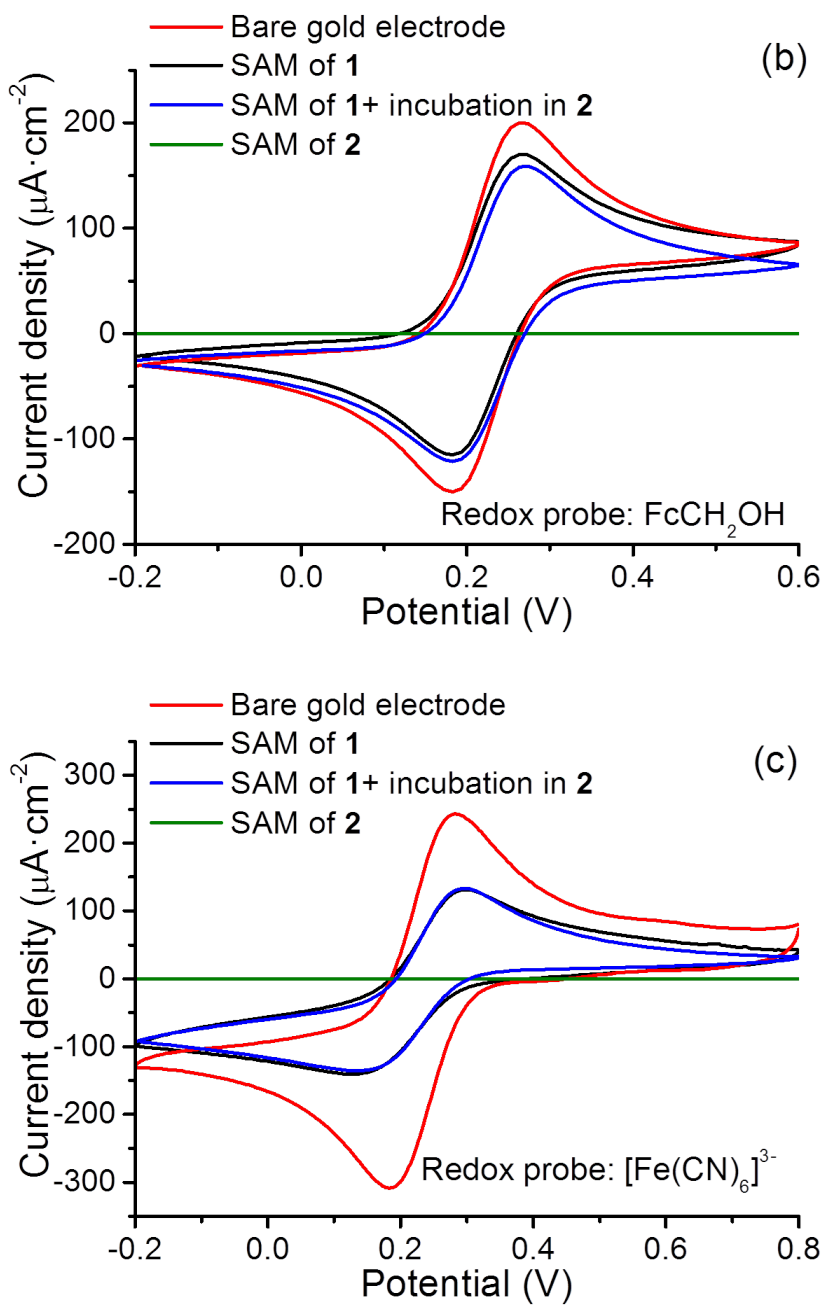

Figure 2. Cyclic voltammograms in aqueous $0.1 \mathrm{M} \mathrm{KCl}$ (vs. $\mathrm{Ag} / \mathrm{AgCl}, \mathrm{KCl}(3 \mathrm{M})$ ) for the indicated redox probes of a bare gold electrode, a SAM of 1 deposited onto a gold electrode, a SAM of $\mathbf{1}$ deposited onto a gold electrode and further incubated in a solution of 2 for $24 \mathrm{~h}$, a SAM of 2 . In all cases the scan rate was $100 \mathrm{mV} \cdot \mathrm{s}^{-1}$. (a) $1 \mathrm{mM}\left[\mathrm{Ru}\left(\mathrm{NH}_{3}\right)_{6}\right] \mathrm{Cl}_{3}$; (b) $1 \mathrm{mM} \mathrm{FcMeOH}$; (c) $1 \mathrm{mM} \mathrm{K}_{3}\left[\mathrm{Fe}(\mathrm{CN})_{6}\right]$.

Although the high surface coverage of the monolayer determined from the QCM, AFM and STM experiments are indicative of a closely packed monolayer, it could be argued that the electrochemical results in Figure 2 might arise from penetration of the redox probes through the monolayer to the underlying electrode surface. To rule out this possibility, a SAM-modified gold electrode was subsequently incubated in a $1 \cdot 10^{-3} \mathrm{M}$ solution of octadecanethiol (2) for 24 hours. Thiols strongly chemisorb onto gold substrates and should effectively infill any putative pores or holes in the monolayer of $\mathbf{1}$. However, as shown in Figure 2, the voltammograms of the various redox probes at electrodes modified 
by the SAM of $\mathbf{1}$ incubated in $\mathbf{2}$ are essentially unchanged, i.e., there is no significant inhibition of the electrode response after incubation of the modified electrode in the thiol solution. The resilience of the SAM of $\mathbf{1}$ to replacement by octadecanethiol is in marked contrast to the ready exchange of alkynyl-terminated alkyl ferrocenes and alkylthiols between the respective SAMs on gold and molecules in solution as has been observed by Tucker and Gorman. ${ }^{79}$ It seems probable that the tightly packed monolayer of $\mathbf{1}$ is responsible at least in part for limiting the exchange processes.

Electrochemical capacitance determined from cyclic voltammograms of film-covered electrodes in an inactive base electrolyte in the electrochemical "double-layer" region (i.e. where no faradaic current flows) can provide an indication of the macroscopic permeability of the SAM, with permeable or significantly defective films generally showing elevated capacitance. Such voltammograms were obtained for a bare gold and a gold electrode covered by the SAM of $\mathbf{1}$. Cyclic voltammograms recorded at high scan rates $\left(1 \mathrm{~V} \cdot \mathrm{s}^{-1}\right)$ in the window potential from $-0.1 \mathrm{~V}$ to $-0.6 \mathrm{~V}$ in $0.5 \mathrm{M} \mathrm{KCl}$ were obtained (Figure 3). The double layer capacitance noticeably decreased from $35.0 \mu \mathrm{F} \cdot \mathrm{cm}^{-2}$ for the bare gold electrode to $5.2 \mu \mathrm{F} \cdot \mathrm{cm}^{-2}$ for the gold electrode covered by the SAM of $1{ }^{80}$ The low value of capacitance in the latter case points to deposition of a contiguous film on top of the gold substrate. In addition, the response of the monolayer of 1 under these experimental conditions remains unaltered for at least 500 cycles (Figure S13), demonstrating the stability and robustness of the film.

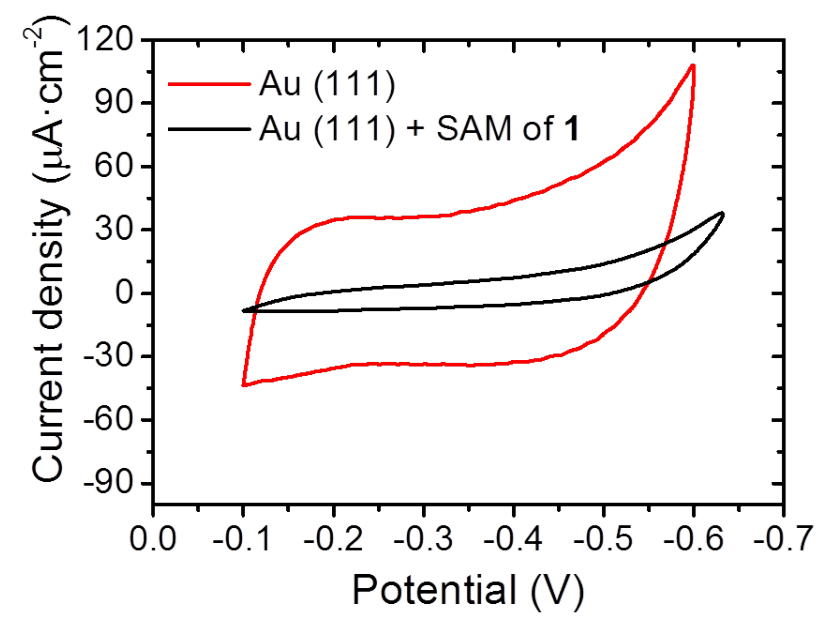

Figure 3. Cyclic voltammograms (first scan) for a gold electrode (red) and a gold electrode covered by a SAM of $\mathbf{1}$ (black). 
The evidence above supports the notion of the monolayer being nearly transparent to the electron transfer to the outer-sphere redox probe on the timescale of these voltammetric measurements. The ability of the Au-C bound monolayer to impede the normal surface electrochemistry of the gold electrode was also investigated by cyclic voltammetry. For the comparison here the electrochemical behavior of the bare and modified gold substrate was analyzed. In aqueous $0.1 \mathrm{M} \mathrm{NaOH}$ the unmodified $\mathrm{Au}(111)$ electrode gives rise to a voltammogram which exhibits sharp peaks at 0.13 and $0.33 \mathrm{~V}$. These waves are characteristic of electrochemical processes of surface adsorbed species, and can be attributed to the potential induced adsorption of $\mathrm{Cl}^{-}$ions (from the $\mathrm{Ag} / \mathrm{AgCl}$ reference electrode) on the gold (gold oxide) electrode (peak A1 in Figure 4, red voltammogram), and adsorption of $\mathrm{OH}^{-}$ions and oxidation of the $\mathrm{Au}(111)$ surface with formation of gold surface oxide (peak A2 in Figure 4, red voltammogram) in excellent agreement with previous observations. $^{47,81,82}$ The subsequent electroreduction of the gold surface oxide is apparent in the cathodic scan (peak $\mathrm{C}$ in Figure 4, red voltammogram). A modified Au(111) electrode covered by a SAM of 1 does not exhibit the peak corresponding to the gold oxide formation (Figure 4, black voltammogram) and only a small wave corresponding to the reduction of the native gold oxide layer is detected on the cathodic scan. These observations are again consistent with inhibition of the gold oxidation process by the SAM of 1. Since it is apparent that gold surface oxidation is shifted by $\sim 100-200 \mathrm{mV}$ in the presence of the SAM this implies very few defects/pinholes in the monolayer. The SAM of 1 therefore protects the gold surface, blocking access of the water molecules and $\mathrm{OH}^{-}$ions required to form the gold oxide, and only after an elevated additional overpotential does surface oxidation occur. Similar inhibition of gold surface oxidation has been reported by Krings et al. for SAMs of thiolated aromatic and $\pi$-conjugated moieties on $\mathrm{Au}(111) .{ }^{83}$ 


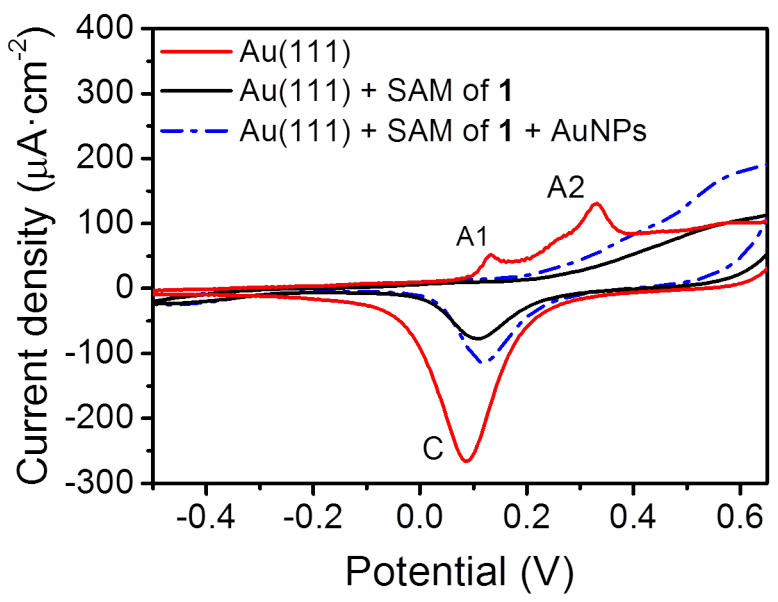

Figure 4. Cyclic voltammograms corresponding to the oxidation of the gold surface on bare $\mathrm{Au}(111)$ or the indicated modified electrodes recorded in aqueous $0.1 \mathrm{M} \mathrm{NaOH}$ at 0.1 $\mathrm{V} \cdot \mathrm{s}^{-1} \mathrm{vs} \mathrm{Ag} / \mathrm{AgCl}$.

As the SAM of $\mathbf{1}$ is acetylene terminated, this provides a reactive moiety for further chemistry on the exposed 'top' surface. This is demonstrated here through the attachment of gold nanoparticles on top of the monolayer. These nanoparticles then also exhibit voltammograms the shape of which demonstrate electronic communication with the underlying gold substrate through the monolayer of $\mathbf{1}$, and further attests to the electronic transparency of the monolayer. For this voltammetric analysis a SAM of $\mathbf{1}$ was deposited on an $\mathrm{Au}(111)$ substrate and it was further modified by incubation for 3 hours in an aqueous solution of ligand free gold nanoparticles (AuNPs) following a previously reported procedure, ${ }^{42}$ which is comprehensively described in the SI (Figure S14). Voltammograms recorded using the resulting AuNPs decorated SAM in aqueous $0.1 \mathrm{M} \mathrm{NaOH}$ (indicated in blue, Figure 4) exhibit a broad, featureless anodic profile, with the additional current corresponding to the electrochemical response of polycrystalline AuNPs. ${ }^{84,85}$ An enhanced electroreduction peak is also observed following adsorption of AuNPs on-top of the monolayer and this is attributed to the reduction of the electrochemically formed oxide on the nanoparticles. In other words, the additional current response between the blue (monolayer of $\mathbf{1}+$ AuNPs) and black (monolayer of $\mathbf{1}$ only) voltammograms is not the result of diffusion of $\mathrm{OH}^{-}$ions and water molecules through putative defects or pinholes in the organic layer, but it is associated with the electrochemical response of the attached 
AuNPs. Therefore, these sets of experiments reveal that the compact SAM of $\mathbf{1}$ formed onto the gold electrode acts as a conduit for charge transfer to the outlying gold nanoparticles. These observations together (Figures 3 and 4) are indicative of a compact monolayer of 1 that efficiently permits electron transfer through the SAM to redox couples approaching from solution.

Previous studies ${ }^{47}$ have also used cyclic voltammetry to assess the electrochemical stability of the SAMs by sweeping to anodic and cathodic potentials, respectively. The onset of oxidative or reductive currents are then used to define stability limits. For this purpose cyclic voltammograms of SAMs of 1 are recorded here in both $0.1 \mathrm{M} \mathrm{HClO}_{4}$ and $0.1 \mathrm{M} \mathrm{NaOH}$. In $0.1 \mathrm{M} \mathrm{HClO}_{4}$ a SAM of $\mathbf{1}$ is electrochemically inert (only a constant capacitive charging current is detected) until about $1 \mathrm{~V}$. At this potential a large faradaic oxidation wave starts to appear, peaking at $\sim 1.4 \mathrm{~V}$, black voltammogram in Figure 5 . The electrochemical charge involved in this oxidation wave on the initial cycle is related to both the oxidative desorption of $\mathbf{1}$ and to contributions arising from the formation of the gold oxide monolayer. Indeed, the onset for the formation of the gold oxide monolayer appears at more positive potentials than on a bare $\mathrm{Au}(111)$ substrate, red voltammogram in Figure 5 , included as a control experiment (red voltammogram, Figure 5). The charge corresponding to this broad anodic peak amounts to $1890 \mu \mathrm{C} \cdot \mathrm{cm}^{-2}$. The peak current decreases on repetitive cycling of the voltammogram, until only a wave with consistent peak current corresponding to gold oxide formation is observed (blue plot in Figure 5), which is almost identical in on-set voltage and current to a bare $\mathrm{Au}(111)$ electrode of the same dimensions. From this it is concluded that the oxidative desorption of $\mathbf{1}$ does not foul the $\mathrm{Au}(111)$ surface. The anodic scan exhibits now an oxidation charge of $826 \mu \mathrm{C} \cdot \mathrm{cm}^{-2}$ which is characteristic of a bare $\operatorname{Au}(111)$ electrode with a certain density of surface defects. ${ }^{86}$ After repetitive cycling to these anodic limits, no electrochemical desorption peak could be subsequently detected in $\mathrm{NaOH} 0.1 \mathrm{M}$ at cathodic potentials. From this it is deduced that the monolayer of $\mathbf{1}$ has been oxidatively desorbed from the $\mathrm{Au}(111)$ surface. In addition, by integrating the electrochemical charge involved in the oxidative electrodesorption peak, (black voltammogram in Figure 5), and by subtracting the anodic charge registered in the later scan (blue voltammogram in Figure 5), a value of 1064 $\mu \mathrm{C} \cdot \mathrm{cm}^{-2}$ is obtained. Whilst the mechanism of oxidative desorption has not been explored 
in detail, this experiment serves to set the oxidative limit of the electrochemical window of the SAM of $\mathbf{1}$ on $\mathrm{Au}(111)$. Reductive desorption $\mathrm{CV}$ experiments of a SAM of $\mathbf{1}$ on an $\mathrm{Au}(111)$ electrode in $0.1 \mathrm{M} \mathrm{NaOH}$ are characterized by a large cathodic peak at $-1.25 \mathrm{~V}$ vs. $\mathrm{Ag} / \mathrm{AgCl}$ (Figure S15) with an associated faradaic charge of $-1.23 \mathrm{mC} \cdot \mathrm{cm}^{-2}$. This faradaic charge is significantly higher than the expectation for an exclusively one-electron electrochemical desorption of $\mathbf{1}$ (as estimated from the electrode area and SAM packing density as a maximum charge of about $-0.08 \mathrm{mC} \cdot \mathrm{cm}^{-2}$ ). This large reductive faradaic charge can be attributed to a combination of the electrodesorptive reduction of $\mathbf{1}$ and the hydrogen evolution reaction (HER). ${ }^{87}$ The onset of the oxidation current is $1 \mathrm{~V}$ (in $0.1 \mathrm{M} \mathrm{HClO}_{4}$ ) while the onset of the cathodic currents is around $0.9 \mathrm{~V}$ (in $0.1 \mathrm{M} \mathrm{NaOH}$ ), giving a "potential window" of $1.9 \mathrm{~V}$ between these two current onset values. On the other hand, other literature work by $\mathrm{Fu}$ et $\mathrm{al}^{44}$ on the stability of ethynyl-terminated oligo(phenylene ethynylene)-based moieties assembled on $\mathrm{Au}(111)$ formed using desilylation chemistry defined the stability potential window by using peak currents rather than onset currents. Although, in our opinion stability is better defined by using onset current rather than peak currents, the use of the latter does provide a direct comparison between our work and that of $\mathrm{Fu}$ et al. Using peak currents we define a potential window of $2.6 \mathrm{~V}$ for monolayers of 1 which compares favorably with the potential window of $\sim 2.2 \mathrm{~V}$ reported by Fu et $a l .{ }^{44}$ for other ethynyl-terminated oligo(phenylene ethynylene)-based moieties assembled on $\mathrm{Au}(111)$.

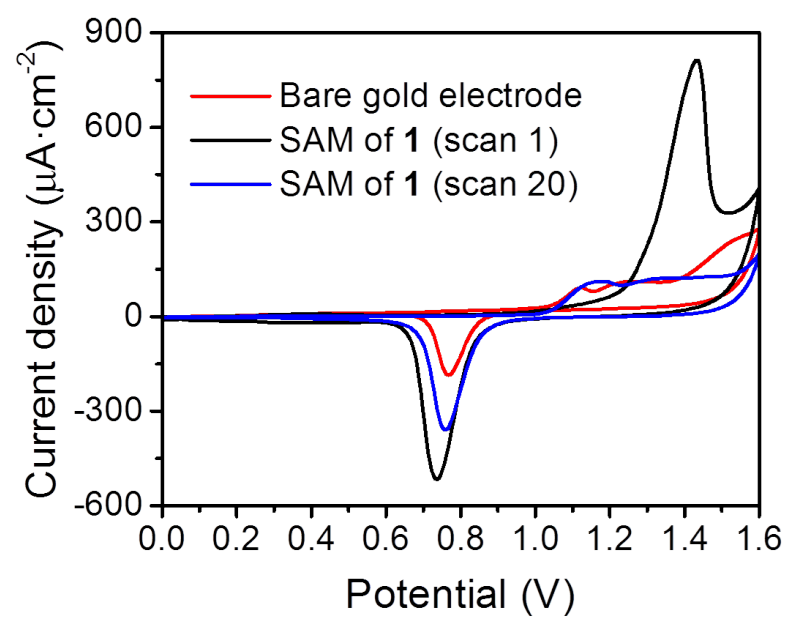

Figure 5. Cyclic voltammograms for the electrochemical desorption of the SAM of 1, recorded at $0.05 \mathrm{~V} \cdot \mathrm{s}^{-1}$ in aqueous $0.1 \mathrm{M} \mathrm{HClO}_{4}$. 
Following on from these electrochemical determinations of the facile electrontransfer characteristics of the SAM of 1 on gold, the electrical properties of these monolayers within electrode|SAM|electrode molecular junctions can also be analyzed directly. It is well known that the charge transport mechanism in molecular junctions of a series of $\pi$-conjugated molecules similar to 1 undergoes a transition from direct tunneling (rectangular barrier) to field emission (triangular barrier) upon increasing the applied bias. $^{88,89}$ It is usually assumed that the voltage at which this transition occurs ( $\left.V_{\text {trans }}\right)$ is linearly correlated with the energy offset between the electrode Fermi level and the frontier molecular orbital (FMO) primarily responsible for the conduction in a single channel tunneling model. ${ }^{90}$ Although the transition voltage is not directly equal to this energy offset, it nevertheless does allow us to qualitatively gauge the effective energy barrier to charge transport by measuring $V_{\text {trans. }}$. In other words a high transition voltage would be expected to indicate a high energy offset between the FMO and the Fermi energy, and vice versa. Thus, $V_{\text {trans }}$ is a measurable quantity which allows us to qualitatively prove the molecular orbitalFermi level barrier height without the assumptions and parameter fitting inherent in the Simmons model. This is especially important as the tunnel barrier height is sensitive to the molecular electronic topology, electrode-molecule linking group and electrode type.

To obtain $V_{\text {trans, }}$ a series of $I-V$ curves were recorded with a scanning tunneling microscope (STM) using the "STM touch-to-contact" method (Figure S16) ${ }^{56}$ From set point parameters $U_{t}=0.6 \mathrm{~V}$ (tip bias) and $I_{0}=0.33 \mathrm{nA}$ (set point current) a tip-substrate distance of $1.7 \mathrm{~nm}(s)$ was obtained, which is in agreement with the monolayer thickness, indicating that the STM is positioned immediately above and just touching the SAM. Under these conditions, more than $400 I-V$ curves for a SAM of 1 were recorded; the average $I-V$ curve from all the 400 individual registered curves is shown in the inset of Figure 6. A Fowler-Nordheim plot $\left(\ln \left(I / V^{2}\right)\right.$ against $\left.1 / V\right)$ using this average $I-V$ curve was constructed (Figure 6). ${ }^{56,90}$ A remarkably low value of $0.51 \pm 0.05 \mathrm{~V}$ for $V_{\text {trans }}$ is obtained for the SAM of 1 which is indicative of a relatively small offset between the gold Fermi level and (presumably) the highest occupied molecular orbital (HOMO) of 1. ${ }^{47,88,89,91}$ In order to have a broader picture of the role played by the alkynyl anchor group as well as the OPE structure in the offset between the FMO and the electrode Fermi energy, $V_{\text {trans }}$ for the SAM of 1 is here compared with (i) $V_{\text {trans }}$ values of 'three ring' OPE derivatives containing other 
anchoring groups (Table I); (ii) $V_{\text {trans }}$ values of another conjugated molecular wires also containing alkynyl terminal groups (Table I); (iii) $V_{\text {trans }}$ values obtained for compounds containing OPE skeletons or other highly conjugated molecular moieties (Table S1 in the SI). The $V_{\text {trans }}$ values shown in these Tables show a significantly lower value for $V_{\text {trans }}$ in compound 1 , in comparison with identical 'three ring' OPE structures having other functional groups (amines, thiols). They also demonstrate a lower value for $V_{\text {trans }}$ in compound 1 when compared to other alkynyl terminal groups attached to other conjugated chemical structures. Finally, the combined OPE-alkynyl structure also results in lower $V_{\text {trans }}$ values than those previously reported in the literature for other organic materials. These results support the deductions that the use of the alkynyl moiety directly linked to the OPE structure leads to a lower tunnel barrier and is consistent with the enhanced electronic transport observed through $\mathbf{1}$.

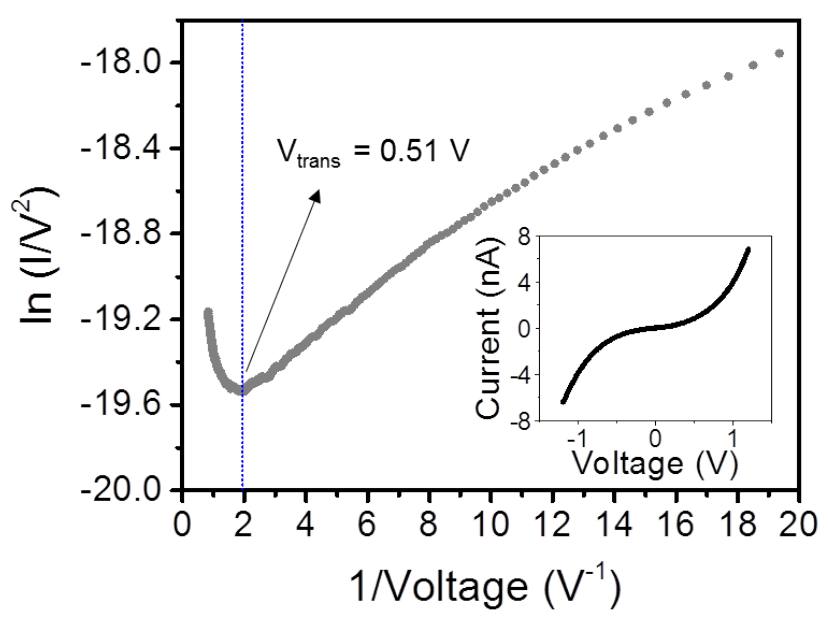

Figure 6. Fowler-Nordheim plot for a SAM of 1 obtained from the inset average $I-V$ curve. The dashed vertical line corresponds to the voltage at which the tunneling barrier transition occurs $\left(V_{\text {trans }}\right)$.

Table I. $\mathrm{V}_{\text {trans }}$ values for the indicated compounds.

\begin{tabular}{|c|c|c|}
\hline & $\mathrm{V}_{\text {trans }}(\mathrm{V})$ & Reference \\
\hline
\end{tabular}




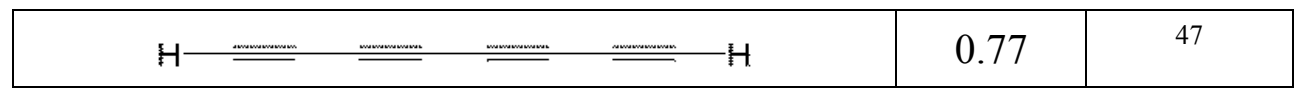

\section{Conclusions}

A complete set of experimental data that include QCM, AFM, STM and CV have clearly demonstrated that SAMs of $\mathbf{1}$ are tightly packed. These monolayers exhibit a surface coverage of $0.97 \times 10^{-9} \mathrm{~mol} \cdot \mathrm{cm}^{-2}$ and are nearly free of defects, holes or three-dimensional aggregates. Additionally, the cyclic voltammetric response exhibited by a bare gold electrode and a gold electrode modified by a SAM of $\mathbf{1}$ for the $\left[\mathrm{Ru}\left(\mathrm{NH}_{3}\right)_{6}\right]^{3+}$ redox probe appear to be almost indistinguishable (neither a marked decrease of the faradaic current density nor increase of the peak potential separation were observed). Together, these results indicate a negligible contribution of defects and pinholes to the faradaic current and point to effective electron transfer through the organic layer. Transition voltage spectroscopy (TVS) analysis further supports an enhanced electronic transport through the SAM of $\mathbf{1}$ when compared to similar OPE SAMs terminated with more conventional surface anchoring groups. Importantly, gold electrodes modified with a SAM of $\mathbf{1}$ also feature a wide electrochemical stability window of some $2.6 \mathrm{~V}$ (measured between oxidative and reductive peaks). The facile formation of alkynyl $\mathrm{C}$-Au contacts and remarkably good ET characteristics of the resulting Au-C contacted SAMs offer new avenues for the fabrication of highly conductive large-area monomolecular films. Key attributes here are the compact surface coverage with low apparent defect density and efficient electron transfer through the organic monolayers formed on a gold electrode. These monolayer films could more generally also offer new approaches for the capping and stabilization of cluster and metal nanoparticles, formation of large 3D NPs scaffolds (by linking together metal nanoparticles through molecular wires strongly linked by $\mathrm{C}$-Au bonds), as well as surface modification of clusters and NPs to tune physical and optical properties, with future applications in the fabrication of electrochemical sensors and immunosensors, plasmonics, electrocatalysis, electroanalysis, and photovoltaics.

\section{Acknowledgements}

P.C. and J.L.S. are grateful for financial assistance from Ministerio de Economia y Competitividad from Spain and fondos FEDER in the framework of projects MAT201678257-R and CTQ2015-70174-P, respectively. J.L.S. also acknowledges the funded project 
Hierarchical Self Assembly of Polymeric Soft Systems, "SASSYPOL", from the 7th Framework Programme (CEE, Ref-607602). L.H., S.M., J.L.S, and P.C. acknowledge support from DGA/Fondos FEDER (construyendo Europa desde Aragón) for funding PLATON (E31_17R) and CLIP (E47_17R) research groups. R.J.N. thanks EPSRC for funding (EP/M029522/1, EP/K007785/1, EP/M014169/1 and EP/M005046/1), and P.J.L. also gratefully acknowledges support from the Australian Research Council (FT120100073; DP140100855). Authors also thank Marta Lafuente from the Institute of Nanoscience of Aragon for the preparation of the SERS substrates.

\section{References}

1. N. Kobayashi, Y. Kamei, Y. Shichibu, K. Konishi, J. Am. Chem. Soc., 2013, 135, 16078.

2. P. Maity, S. Takano, S. Yamazoe, T. Wakabayashi, T. Tsukuda, J. Am. Chem. Soc., 2013, 135, 9450 .

3. C. Jia, X. Guo, Chem. Soc. Rev., 2013, 42, 5642.

4. K.-K. Wan, W. W. Xu, S.-F. Yuan, Y. Gao, X.-C. Zeng, W.-M. Wang, Angw. Chem. Int. Ed., 2015, 54, 9683.

5. X.-k. Wan, W. W. Xu, S.-f. Yuan, Y. Gao, X.-c. Zeng, M. Wang, 2015, 24, 9683.

6. Y. Wang, X.-k. Wan, L. Ren, H. Su, G. Li, S. Malola, S. Lin, Z. Tang, B. K. Teo, Q.-m. Wang, N. Zheng, J. Am. Chem. Soc., 2016, 138, 3278.

7. Z. Lei, X.-k. Wan, S.-f. Yuan, J.-q. Wang, Dalton Trans., 2017, 46, 3427.

8. A. Vilan, D. Aswal, D. Cahen, Chem. Rev., 2017, 117, 4248.

9. S.-S. Zhang, H.-F. Su, G.-L. Zhuang, X.-P. Wang, C.-H. Tung, D. Sun, L.-S. Zheng, Chem. Commun., 2018, 54, 11905.

10. L. J. Richter, C. S.-C. Yang, P. T. Wilson, C. A. Hacker, R. D. van Zee, J. J. Stapleton, D. L. Allara, Y. Yao, J. M. Tour, J. Phys. Chem. B, 2004, 108, 12547.

11. H. Häkkinen, Nat. Chem., 2012, 4, 443.

12. A. Villares, D. P. Lydon, P. J. Low, B. J. Robinson, G. J. Ashwell, F. M. Royo, P. Cea, Chem. Mater., 2008, 20, 258.

13. S. Martin, W. Haiss, S. Higgins, P. Cea, M. C. Lopez, R. J. Nichols, J. Phys. Chem. C, 2008, 112, 3941. 
14. L. M. Ballesteros, S. Martín, J. Cortés, S. Marqués-González, S. J. Higgins, R. J. Nichols, P. J. Low, P. Cea, Chem. Eur. J., 2013, 19, 5352.

15. Y. Xing, T. H. Park, R. Venkatramani, S. Keinan, D. N. Beratan, M. J. Therien, E. Borguet, J. Am. Chem. Soc., 2010, 132, 7946.

16. A. Villares, D. P. Lydon, B. J. Robinson, G. Ashwell, F. M. Royo, P. J. Low, P. Cea, Surf. Sci., 2008, 602, 3683.

17. B. Q. Xu, N. J. Tao, Science, 2003, 301, 1221.

18. S. Y. Quek, M. Kamenetska, M. L. Stigerwald, H. J. Choi, S. G. Louie, M. S. Hybertsen, J. B. Neaton, L. Venkataraman, Nat. Nanotechnol., 2009, 4, 230.

19. W. Hong, H. Li, S.-X. Liu, Y. Fu, J. Li, V. Kaliginedi, S. Decurtins, T. Wandlowski, J. Am. Chem. Soc., 2012, 134, 19425.

20. H. M. Osorio, S. Martin, M. Carmen Lopez, S. Marques-Gonzalez, S. J. Higgins, R. J. Nichols, P. J. Low, P. Cea, Beilstein Nanotechnol., 2015, 6, 1145.

21. L. A. Zotti, T. Kirchner, J. C. Cuevas, F. Pauly, T. Huhn, E. Scheer, A. Erbe, Small, $2010,6,1529$.

22. A. Mishchenko, L. A. Zotti, D. Vonlanthen, M. Bulrkle, F. Pauly, J. C. Cuevas, M. Mayor, T. Wandlowski, J. Am. Chem. Soc., 2011, 133, 184.

23. L. Venkataraman, J. E. Klare, I. W. Tam, C. Nuckolls, M. S. Hybertsen, M. N. Steigerwald, Nano Lett., 2006, 6, 458.

24. B. Kim, J. M. Beebe, Y. Jun, X. Y. Zhu, C. D. Frisbie, J. Am. Chem. Soc., 2006, 128, 4970.

25. C. Ko, M. Huang, M. Fu, C. Chen, J. Am. Chem. Soc., 2010, 132, 756.

26. Y. S. Park, A. C. Whalley, M. Kamenetska, M. L. Steigerwald, M. S. Hybertsen, C. Nuckolls, L. Venkataraman, J. Am. Chem. Soc. , 2007, 129, 15768.

27. N. Katsonis, A. Marchenko, S. Taillemite, D. Fichou, G. Chouraqui, C. Aubert, M. Malacria, Chem. Eur. J., 2003, 9, 2574.

28. N. Katsonis, A. Marchenko, D. Fichou, N. Barret, Surf. Sci., 2008, 602, 9.

29. A. Nion, N. Katsonis, A. Marchenko, C. Aubert, D. Fichou, New J. Chem., 2013, 37, 2261.

30. E. G. Petrov, A. Marchenko, O. Kapitanchuk, N. Katsonis, D. Fochou, Mol. Cryst. Liq. Crys., 2014, 589, 3. 
31. G. Pera, S. Martín, L. M. Ballesteros, A. J. Hope, P. J. Low, R. J. Nichols, P. Cea, Chem. Eur. J., 2010, 16, 13398.

32. S. Marques-Gonzalez, D. S. Yufit, J. A. K. Howard, S. Martin, H. M. Osorio, V. M. Garcia-Suarez, R. J. Nichols, S. J. Higgins, P. Cea, P. J. Low, Dalton Trans., 2013, 42, 338 .

33. R. R. Ferradás, S. Marqués-González, H. M. Osorio, J. Ferrer, P. Cea, D. C. Milan, A. Vezzoli, S. J. Higgins, R. J. Nichols, P. J. Low, V. M. García-Suárez, S. Santiago Martín, RSC Adv., 2016, 6, 75111.

34. H. M. Osorio, S. Martin, D. C. Milan, A. Gonzalez-Orive, J. B. G. Gluyas, S. J. Higgins, P. J. Low, R. J. Nichols, P. Cea, J. Mater. Chem. C, 2017, 5, 11717.

35. J. Fock, J. K. Sorensen, E. Lortscher, T. Vosch, C. A. Martin, H. Riel, K. Kilsa, T. Bjornholm, H. van der Zant, Phys. Chem. Chem. Phys., 2011, 13, 14325.

36. G. K. Ramachandran, T. J. Hopson, A. M. Rawlett, L. A. Nagahara, A. Primak, S. M. Lindsay, Science, 2003, 300, 1413.

37. Z. K. Keane, J. W. Ciszek, J. M. Tour, D. Natelson, Nano Lett., 2006, 6, 1518.

38. S. Yasuda, S. Yoshida, J. Sasaki, Y. Okutsu, T. Nakamura, A. Taninaka, O.

Takeuchi, H. Shigekawa, J. Am. Chem. Soc., 2006, 128, 7746.

39. D. Vuillaume, Proc. IEEE, 2010, 98, 2111.

40. Z. L. Cheng, R. Skouta, H. Vazquez, J. R. Widawsky, S. Schneebeli, W. Chen, M.

S. Hybertsen, R. Breslow, L. Venkataraman, Nat. Nanotechnol., 2011, 6, 353.

41. T. Hines, I. Díez-Pérez, H. Nakamura, T. Shimazaki, Y. Asai, N. Tao, J. Am. Chem. Soc., 2013, 135, 3319.

42. H. M. Osorio, P. Cea, L. M. Ballesteros, I. Gascon, S. Marqués-González, R. J. Nichols, F. Pérez-Murano, P. J. Low, S. Martín, J. Mater. Chem. C., 2014, 2, 7348.

43. F. Bejarano, Olavarria-Contreras, A. Droghetti, I. Rungger, A. Rudnev, D. Gutiérrez, M. Mas-Torrent, J. Veciana, H. van der Zant, C. Rovira, E. Burzurí, N. Crivilliers, J. Am. Chem. Soc., 2018, 140, 1691.

44. Y. Fu, S. Chen, A. Kuzume, A. Rudnev, C. Huang, V. Kaliginedi, M. Baghernejad, Q. J. Hong, T. Wandlowski, S. Decurtins, S.-X. Liu, Nat Commun, 2015, 6, 6403.

45. J. Liang, R. E. G. Smith, A. Vezzoli, L. Xie, D. C. Milan, R. Davidson, A. Beeby, P. J. Low, S. J. Higgins, B. Mao, R. J. Nichols, Electrochim. Acta, 2016, 220, 436. 
46. W. Hong, D. Z. Manrique, P. Moreno-Garcia, M. Gulcur, A. Mishchenko, C. J. Lambert, M. R. Bryce, T. Wandlowski, J. Am. Chem. Soc., 2012, 134, 19425.

47. A. Moneo, A. González-Orive, S. Bock, M. Fenero, I. L. Herrer, D. C. Milan, M. Lorenzoni, R. J. Nichols, P. Cea, F. Perez-Murano, P. J. Low, S. Martin, Nanoscale, 2018, 10, 14128.

48. W. Chen, J. R. Widawsky, H. Vazquez, S. Schneebeli, M. S. Hybertsen, R. Breslow, L. Venkataraman, J. Am. Chem. Soc., 2011, 133, 17160.

49. J. R. Widawsky, W. Chen, H. Vázquez, T. W. Kim, R. Breslow, M. S. Hybertsen, L. Venkataraman, Nano Lett., 2013, 13, 2889.

50. D. Coulson, Inorg. Synth., 1990, 28, 107.

51. W. M. Khairul, L. Porres, D. Albesa-Jove, M. S. Senn, M. G. Jones, D. P. Lydon, J. A. K. Howard, A. Beeby, T. B. Marder, P. J. Low, J. Cluster. Sci., 2006, 17, 65.

52. A. Coskun, E. U. Akkaya, J. Am. Chem. Soc., 2006, 128, 14474.

53. A. M. McDonagh, H. M. Zareie, M. J. Ford, C. S. Barton, M. Ginic-Markovic, J. G. Matisons, J. Am. Chem. Soc., 2007, 129, 3533.

54. T. Zaba, A. Noworolska, C. M. Bowers, B. Breiten, G. M. Whitesides, P. Cyganik, J. Am. Chem. Soc., 2014, 136, 11918.

55. M. Lafuente, I. Pellejero, V. Sebastián, M. A. Urbiztondo, R. Mallada, M. P. Pina, J. Santamaría, Sensors and Actuators B: Chemical, 2018, 267, 457.

56. L. M. Ballesteros, S. Martin, S. Marqués-González, M. C. López, S. Higgins, R. J. Nichols, P. J. Low, P. Cea, J. Phys. Chem. C., 2015, 119, 784.

57. W. Haiss, D. Lackey, J. K. Sass, J. Chem. Phys., 1991, 95, 2193.

58. K. Liu, G. Li, X. Wang, F. Wang, J. Phys. Chem. C, 2008, 112, 4342.

59. C. D. Bain, G. M. Whitesides, J. Phys. Chem., 1989, 93, 1670.

60. P. Cea, S. Martín, A. González-Orive, H. M. Osorio, P. Quintín, L. Herrer, J. Chem. Ed., 2016, 93, 1441.

61. L. M. Ballesteros, S. Martín, C. Momblona, S. Marqués-González, M. C. López, R. J. Nichols, P. J. Low, P. Cea, J. Phys. Chem. C., 2012, 116, 9142.

62. F. Anariba, S. H. DuVall, R. L. McCreery, Anal. Chem., 2003, 75, 3837.

63. S. Y. Sayed, A. Bayat, M. Kondratenko, Y. Leroux, P. Hapiot, R. L. McCreery, J. Am. Chem. Soc., 2013, 135, 12972. 
64. L. Laurentius, S. R. Stoyanov, S. Guserov, A. Kovalenko, R. Du, G. P. Lopinski, M. T. McDermott, ACS Nano, 2011, 5, 4219.

65. S. Zhang, K. L. Chandra, C. B. Gorman, J. Am. Chem. Soc., 2007, 129, 4876.

66. M. J. Ford, R. C. Hoft, A. McDonagh, J. Phys. Chem. B., 2005, 20387.

67. O. Chailapakul, R. M. Crooks, Langmuir, 1995, 11, 1329.

68. R. L. McCreery, Chem. Rev., 2008, 108, 2646.

69. S. Tanimoto, A. Ichimura, J. Chem. Edu., 2013, 90, 778.

70. P. H. Chen, M. A. Fryling, R. L. Mccreery, Anal. Chem., 1995, 3115.

71. A. J. Bard, L. R. Faulkaner, Electrochemical methods: fundamentals and applications. (Wiley, New York, ed. 2nd, 2001).

72. M. Fleischmann, P. R. Graves, J. Robinson, J. Electroanal. Chem., 1985, 182, 87.

73. S. N. Ovchinnikova, Russian Journal of Electrochemistry, 2017, 56, 1246.

74. T. Felgenhauer, H. T. Rong, M. Buck, J. Electroanal. Chem., 2003, 550-551, 309.

75. M. C. R. González, A. G. Orive, P. Carro, R. C. Salvarezza, A. H. Creus, J. Phys. Chem. C, 2014, 118, 30013.

76. A. Villares, G. Pera, S. Martín, R. J. Nichols, D. P. Lydon, L. Applegarth, A.

Beeby, P. J. Low, P. Cea, Chem. Mater., 2010, 22, 2041.

77. L. M. Ballesteros, S. Martín, G. Pera, P. A. Schauer, N. J. Kay, M. C. López, P. J. Low, R. J. Nichols, P. Cea, Langmuir, 2011, 27, 3600.

78. L. M. Ballesteros, S. Martin, G. Pera, P. A. Schauer, N. J. Kay, M. Carmen Lopez, P. J. Low, R. J. Nichols, P. Cea, Langmuir, 2011, 27, 3600.

79. C. B. Gorman, R. L. Carroll, R. R. Fuierer, Langmuir, 2001, 17, 6923.

80. C. Miller, P. Cuendet, Grätzel, J. Phys. Chem., 1991, 95, 877.

81. A. Hamelin, M. J. Sottomayor, F. Silva, S. C. Chang, M. J. Weaver, J. Electroanal. Chem., 1990, 295, 291.

82. B. Wurster, D. Grumelli, D. Hötger, R. Gutzler, K. Kern, J. Am. Chem. Soc., 2016, 138, 3623.

83. N. Krings, H. H. Strehblow, J. Kohnert, H. D. Martin, Electrochim. Acta, 2003, 1, 167. 
84. A. Orive, D. Grumelli, C. Vericat, J. Ramalo-Lópex, L. Giovanetti, G. Benitez, J. Azcarate, G. Corthey, M. Fonticelli, F. Requejo, A. Hernández Creus, R. C.

Salvarezza, Nanoscale, 2011, 3, 1708.

85. C. Gutiérrez-Sánchez, M. Pita, C. Vaz-Domínguez, S. Shleev, A. L. De Lacey, J. Am. Chem. Soc., 2012, 134, 17212.

86. S. F. L. Mertens, A. Bütikofer, L. Siffert, T. Wandlowski, Electroanalysis, 2010, 22, 2940 .

87. M. I. Muglali, A. Erbe, Y. Chen, C. Barth, P. Koelsch, M. Rohwerder, Electrochim. Acta, 2013, 90, 17.

88. Q. Lu, K. Liu, H. Zhang, Z. Du, X. Wang, F. Wang, ACS Nano, 2009, 3, 3861.

89. J. M. Beebe, B. Kim, C. D. Frisbie, J. G. Kushmerick, ACS Nano, 2008, $2,827$.

90. J. M. Beebe, B. Kim, J. W. Gadzuk, C. D. Frisbie, J. G. Kushmerick, Phys. Rev. Lett., 2006, 97, 026801.

91. S. H. Choi, B. Kim, C. D. Frisbie, Science, 2008, 320, 1482. 